\title{
A Module-based Approach to Teaching Big data and Cloud Computing Topics at CS Undergraduate Level
}

\author{
Debzani Deb \\ Department of Computer Science \\ Winston-Salem State University \\ Winston-Salem, NC, USA \\ debd@wssu.edu
}

\author{
Muztaba Fuad \\ Department of Computer Science \\ Winston-Salem State University \\ Winston-Salem, NC, USA \\ fuadmo@wssu.edu
}

\author{
Keith Irwin \\ Department of Computer Science \\ Winston-Salem State University \\ Winston-Salem, NC, USA \\ irwinke@wssu.edu
}

\begin{abstract}
Big data and cloud computing collectively offer a paradigm shift in the way businesses are now acquiring, using and managing information technology. This creates the need for every CS student to be equipped with foundational knowledge in this collective paradigm and to possess some hands-on experience in deploying and managing big data applications in the cloud. We argue that, for substantial coverage of big data and cloud computing concepts and skills, the relevant topics need to be integrated into multiple core courses across the undergraduate CS curriculum rather than creating additional standalone core or elective courses and performing a major overhaul of the curriculum. Our approach to including these topics is to develop autonomous learning modules for specific core courses in which their coverage might find an appropriate context. In this paper, three such modules are discussed and our classroom experiences during these interventions are documented. So far, we have achieved reasonable success in attaining student learning outcomes, enhanced engagement, and interests. Our objective is to share our experience with the academics who aim at incorporating similar pedagogy and to receive feedback about our approach.
\end{abstract}

\section{CCS CONCEPTS}

Social and professional topics $\sim$ Computer science education - Social and professional topics Information technology education $\cdot$ Computer systems organization $\sim$ Cloud computing

\section{KEYWORDS}

Big data; Cloud computing; Curriculum; Mapreduce; Apache Spark.

\section{ACM Reference format:}

Debzani Deb, Muztaba Fuad, Keith Irwin. 2019. A Module-based Approach to Teaching Big data and Cloud Computing Topics at CS Undergraduate

Permission to make digital or hard copies of all or part of this work for personal or classroom use is granted without fee provided that copies are not made or distributed for profit or commercial advantage and that copies bear this notice and the full citation on the first page. Copyrights for components of this work owned by others than ACM must be honored. Abstracting with credit is permitted. To copy otherwise, or republish, to post on servers or to redistribute to lists, requires prior specific permission and/or a fee. Request permissions from Permissions@acm.org. SIGCSE '19, February 27-March 2, 2019, Minneapolis, MN, USA

(c) 2019 Association for Computing Machinery.

ACM ISBN 978-1-4503-5890-3/19/02...\$15.00

https://doi.org/10.1145/3287324.3287494
Level. In Proceedings of $50^{\text {th }}$ ACM Technical Symposium on Computer Science Education (SIGCSE '19), February 27-March 2, 2019, Minneapolis, MN, USA. ACM, NY, NY, USA, 7 pages. https://doi.org/10.1145/3287324.3287494

\section{Introduction}

In today's world, the analysis of 'big data' becomes a high priority task for many fields of study and data-driven discovery and decision processes now guide many sectors of our business and economy. At the development level, analyzing big data requires proficiency in specialized algorithms and methodologies due to the fundamentally distributed and parallel nature of the workloads, while, at the infrastructure level, cloud computing skills are paramount to acquire a pool of virtual resources in a 'pay-as-you-go' fashion to deploy and manage these workloads. Big data and cloud computing collectively offer a paradigm shift in the way businesses are now acquiring, using and managing information technology. With the fast growth of this paradigm, businesses are struggling to find experienced people who not only have the deep analytical skills, but also have the data hosting, storage and management skills to effectively leverage this collective model. This observation is supported by the recent prediction, made by the International Data Corporation (IDC), that by 2020 big data staffing shortages will expand from analysts and data scientists to include architects and experts in data management. Increasing adoption of this collective paradigm in solving problems from a variety of domains is also making study and research on this paradigm crucial.

It is immensely important that every CS and IT undergraduate student be equipped with foundational knowledge in this collective paradigm and be provided with hands-on experience in deploying and managing big data applications in the cloud, to acquire skills that are necessary to meet current and future industry demands as well as to enable them to carry out applied research in this paradigm. However, the challenge is that many of the tools and techniques of the big data and cloud computing paradigm have emerged only in the last few years and have not yet transitioned into the most recent ACM/IEEE Joint Curriculum recommendations [1] or the ABET curriculum requirements [2]. Many 2-year and 4-year institutions develop their CS curriculum around these guidelines and requirements and as a result cannot afford to include these contemporary topics within a core course in their densely packed curricula. 
6. Does your department offer courses on emerging PDC topics for undergraduate students? If yes, please choose the most appropriate theme of that course(s) from the following list. Also $\mathrm{c} . . . \mathrm{f}$ it is a required/core course(s) and the level of the course(s).

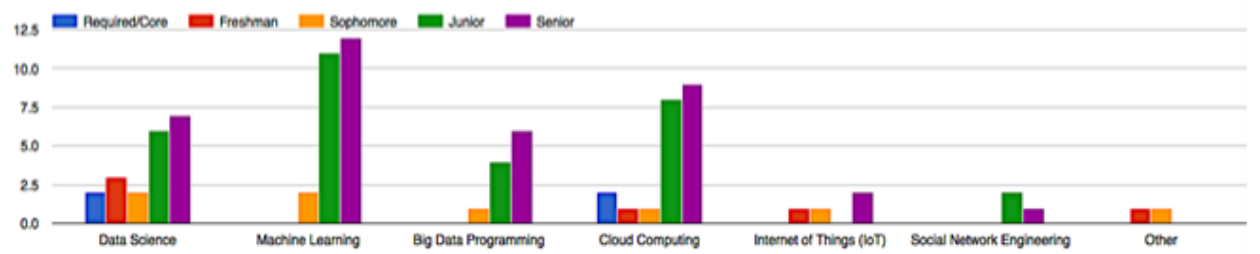

Figure 1: SIGCSE Listserv survey results

We argue that for substantial coverage of big data and cloud computing concepts and skills, students need to be intervened more often, gradually, and the topics should be integrated into multiple core courses of the curriculum. Our approach to including these topics is to develop a series of short, selfcontained learning modules with specific learning goals, lessons plans, and assessment resources, and to suggest specific core courses in which their coverage might find an appropriate context. Each module covers topics of the collective paradigm in the context of a conventional core CS/IT course, thus enabling us to better expose students to the big data and cloud computing concepts without a major overhaul of the curriculum. Providing students with hands-on experience is vital and therefore the modules are designed with a rich set of tutorials, sample programs, descriptions of cloud-based resources and substantial hands-on projects. Reusability and adaptability are other major concerns, and as a result the modules are designed to be autonomous, made available for downloading [3], and detailed enough for easier adoption by other instructors with minimal experience. This paper details the deployment of the modules in specific courses at our institution, however, they could be adapted for deployment in some other courses with minimal effort.

In this paper, we report on our experiences in offering three such modules in respective courses. More specifically, we discuss the learning outcomes, module content, assessment instruments, and student assessment and survey results. Our objective is to share our experience with the instructors who aim at incorporating similar pedagogy that enhance student knowledge on this collective paradigm. The rest of the paper is organized as follows: Section 2 discusses the related initiatives for including big data and cloud computing topics into the curriculum, section 3 details the modules and their classroom deployments and section 4 concludes the paper.

\section{Related Works}

Limited by the fact that there are no standard guidelines provided by ACM or ABET to integrate the big data and cloud computing topics into the CS curriculum, the computer science education community has taken multiple approaches to address the need to produce a satisfactory number of well-trained professionals in these fields. Our extensive literature survey identifies three such approaches from Academia. Firstly, several institutions offer non-core specialized courses $[4,5,6]$ to cover a variety of aspects of data science and big data analytics, where students are primarily taught data acquisition, cleaning, analytical and visualization skills. While these courses help students in developing skills related to transforming data into knowledge, they do not provide the students with concepts and experiences related to hosting, storing, and deploying application within the cloud environment and scaling up applications within performance and budgetary constraints. A second category of approaches integrates big data and/or cloud computing topics in existing CS/IT courses (mostly non-core) such as Parallel and Distributed Computing, High Performance Computing, Networking, Cybersecurity etc. These interventions are often sporadic, mostly ignited by the instructor's own interests and experiences and therefore are not capable of garnering a substantial student interest and knowledge on this collective paradigm. A few research-intensive universities offer specialized standalone courses $[7,8,9,10]$ such as "Cloud computing", "Big data management" etc., where the abovementioned collective paradigm is addressed to a greater extent. However, being an elective (non-core) course offered at a handful of universities, only a handful of students receive the benefit.

The abovementioned approaches are limited in intervening a substantial portion of the CS/IT undergraduate student population with necessary expertise of this collective paradigm as they are mostly undertaken via non-core and special topic courses. A survey was recently administered through SIGCSE listserv regarding the CDER curriculum initiative on parallel and distributed computing (PDC) [11] and one of the questions asked the participants about the emerging topic courses that their departments are offering and whether these courses are core (required) or not. Fig. 1 shows the summarized results based on 35 responses. It is important to note that although many different institutions are conducting many specialized courses that are related to big data and cloud computing at the junior or the senior level, very few of them are core courses and therefore a complete and massive adoption of these topics are yet not possible within this framework. There is a big gap between the advances in big data and cloud computing and their inclusion in college-level instructions and this paper aims to address this gap by proposing an alternative module-based approach that covers 
the related concepts through a set of autonomous modules dispersed over several core courses across the curriculum.

\section{Details of the Modules}

The proposed modules are designed to be short and autonomous for easier adoption and as a result, each of them spans two 75-minute class periods and includes specific learning goals, lecture notes, and assessment resources. The assessment resources contain quiz questions, tutorials, sample programs, cloud-based resources, hands-on projects etc. Detailed tutorials are developed for multiple platforms, enabling straightforward adoption at other institutions. The modules are expected to be taught in a lecture-lab setting, where the first class is typically used for discussing the new concepts and techniques, and the second class is used to introduce students to the hands-on project along with the necessary tools and resources they will require to complete the project. The take home project contains multiple tasks and usually has two weeks of deadline.

The first of the three modules is designed to expose students to the cloud computing fundamentals and to provide them with hands-on experience in using the public cloud environment. The module is adopted to the core "Computer Architecture" (CA) course at our institution. It is important to provide context for the module within the typical course materials where it is deployed so that the module is not perceived by the students as an isolated and disruptive topic. In the CA class, this context is established by asking students to execute CPU/IO benchmarking applications (typical CA topics) in the cloud setting. The second module is focused on MapReduce programming and popular cloud analytics engines (such as Hadoop and Spark) and is deployed in the "Analysis of Algorithm" (AA) class. The context is established by allowing students explore the cost vs. performance tradeoff for running analytics applications within the cloud environment. The third module is designed with the aim of illustrating the importance of SQL within various big database management systems (BDBMS) and is deployed within a "Database Management" (DB) course. The students could easily see the connection when they utilize Spark-SQL programs to load and query both structed and unstructured data sets. The following few sections discuss each module along with the student assessment results in the intervened semesters.

\section{1 "Computer Architecture" (CA) Module}

This module is integrated into the "Computer Architecture" class and is designed to expose students to virtualization and cloud computing fundamentals and to provide students with hands-on experiences in using AWS cloud. At our institution, this is a required course for both CS and IT majors and mostly junior and senior students attend the course. Specific learning outcomes are

1. Recognize the key properties, techniques, strengths and challenges of cloud computing (CA-LO1).

2. Develop hands-on experience with AWS web interface for virtual machine (VM) provisioning and management. (CALO2).
3.1.1 Lesson Plan. The first 75-minute class is lecture-based and includes discussion on important cloud computing characteristics such as scalability, on-demand access, measured services, and elasticity. The lecture then explains resource sharing and virtualization while pointing out its important aspects such as migration, timesharing, isolation etc. Finally, the lecture explores the benefits of utilizing cloud services within a business and the challenges associated with adoption, such as data confidentiality, performance unpredictability, etc. The second class mainly comprises a tutorial and a lab session that instructs students the basic provisioning and management of AWS EC2 instances.

3.1.2 Assessment Instruments. Student learning was assessed by utilizing an exam composed of factual questions and a handson project involving AWS EC2 services. The exam includes true/false, multiple choice and analytical questions and focuses on gauging the progress students have made retaining concepts related to virtualization and cloud computing fundamentals. As part of the hands-on project, students are asked to perform the following tasks

Task 1: Create two EC2 instances with different hardware and software configurations.

Task 2: Analyze instance performances by benchmarking them with CPU- and IO-bound applications and determine how performance scales with the different VM types.

Task 3: Represent results of the analysis graphically and report on them critically.

Students are asked to utilize Systester [12] as CPU-bound benchmark, which calculates the $n^{\text {th }}$ digit of $\pi$ using the GaussLegendre algorithm, while experimenting with various values of $n$ such as $128 \mathrm{~K}, 256 \mathrm{~K}, 512 \mathrm{~K}, 1 \mathrm{M}, 2 \mathrm{M}, 8 \mathrm{M}, 16 \mathrm{M}$. Additionally, the project requires the students to use the iozone benchmarking tool from the Phoronix [13] benchmarking suite to assess IO performances. Students set up experiments to benchmark both read and write performances by running iozone for different record sizes $(4 \mathrm{~KB}$ and $64 \mathrm{~KB})$ and file sizes $(512 \mathrm{MB}, 2 \mathrm{~GB}$, and $8 \mathrm{~GB})$. The experiments were repeated for both instances, the results were expressed graphically, and the students were further asked to critically evaluate their performance analysis results.

3.1.3 Assessment Results. This module was deployed in the Spring 2018 offering of the CA class with 29 students in it. Overall, students performed well (mean:13, median:13, standard deviation: 1.34) in answering the exam questions (out of 15). These statistics indicate students' knowledge acquisition on the concerned topics and therefore show the module's effectiveness in attaining the first learning outcome (CA-LO1). Students were able to accomplish the project with $40 \%$ of them achieving A grade, $20 \%$ of them achieving B grade, $15 \%$ of them securing $C$ grade, and $25 \%$ of them attaining $\mathrm{D} / \mathrm{F}$ grades. Even though, all students were comfortable with the AWS web interface for VM management (especially, after the detailed tutorial session) and accomplished Task 1 successfully (CA-LO2), some had trouble correctly executing the benchmarking applications on the VM instances (Task 2), and a group of students faced challenges while comparing and presenting their results and reporting on 
them critically (Task 3). It is clear that the student population being part of two different majors and backgrounds (CS and IT) was an important factor that contributed to the assessment results, and that the module would benefit more if it were offered to a particular major and tailored accordingly. However, we did not have the opportunity at our institution to accommodate that.

\section{2 "Analysis of Algorithm" (AA) Module}

This module is designed for the students of the Algorithm course with the goal of introducing the popular cloud analytics engines such as Hadoop and Spark. This module is targeted only for the CS majors, who have completed CS1, CS2, and a data structure course and are comfortable with and reasonably proficient using programming languages such as Java or Python. The learning outcomes of the module are

1. Recognize the key properties, techniques, strengths and challenges of MapReduce and Spark Framework (AA-LO1).

2. Build scalable applications based on MapReduce programming model using Hadoop and HDFS. (AA-LO2).

3. Develop basic experience with big data analysis on cloud platforms with performance and cost constraints using Spark/Hadoop platforms (AA-LO3).

3.2.1 Lesson Plan. The first 75-minute class is lecture-based and explores parallel computing at the beginning and introduces MapReduce as a framework that can quickly process large data sets by splitting them into individual chunks that are processed in parallel. The basics of MapReduce programming are then covered with example codes. Apache Hadoop, as an implementation of the MapReduce model, is then introduced along with its distributed file system HDFS. The concept of a Hadoop cluster along with the master-worker framework is briefly explored. Lastly, the problems with the classic MapReduce programming is discussed briefly and the need for an in-memory analytic engine such as Apache Spark is emphasized with a brief description of Spark's runtime environment.

The second class, on the other hand, is a lab-based class where the instructor provides some demonstrations and a tutorial for the students to follow. The tutorial provides instructions on running Hadoop through Cloudera's VM [14] on the local machine. It also provides students with step by step instruction of setting up HDFS, compiling and executing a MapReduce application, and retrieving output from HDFS. Lastly, the tutorial introduces students to Chameleon cloud [15] and shows them how to ssh to a particular Chameleon instance (all students are registered and added to our Chameleon project before the tutorial starts). We implemented a Spark on YARN cluster (with HDFS) on the Chameleon Cloud and the compiled Spark application that the students use for experimentation along with the datasets are pre-loaded to that instance. The concerned Spark application finds the trending topics given the Wikipedia page views information [16]. During the lab session, students are taught how to execute a Spark application in Yarn cluster by using the spark-submit command, and how to configure cluster resources for its execution by varying parameters such as --num-executors, --executor-memory, and -executor-cores. Students are also taught to verify the current resource allocation and to check the execution time and other performance metrics of a spark application by using Spark's web user interface (UI).

3.2.2 Assessment Instruments. This module is assessed by utilizing both hands-on project and exam questions. There are four multiple-choice questions and two design questions developed to assess students' comprehension of the Hadoop/Spark framework and the MapReduce programming model. In the design questions, students are asked to write pseudocode of map and reduce functions (or a series of them) for certain cases. The hands-on project, on the other hand, is built on top of the lab session and includes the following tasks:

Task 1: Modify existing MapReduce code to fulfill new requirements.

Task 2: Develop basic MapReduce program from scratch.

Task 3: Explore Spark application performance in the cloud environment through running them with various runtime configuration settings, and to gain some insight about the resource provisioning and the performance vs. cost tradeoff. Students are presented with two Wikipedia data sets (100GB and 200GB) and are provided with two Spark clusters (one-node cluster and two-node cluster, each compute node with 24 cores, 128 GB memory) in the Chameleon testbed. Students are asked to run the trending Wikipedia Spark application with the given two input datasets while trying various configuration setups for both clusters. Although use of Chameleon is free of charge, we introduce a basic cost model to the students (i.e. 1 service unit = 1 core with 1 GB memory) and ask them to compare different cluster configurations and gain some insight about performance vs. cost tradeoff. Students are further asked to write a report detailing their experimental results and their findings along with their supporting arguments.

3.2.3 Assessment Results. The module was deployed in the Spring 2017 offering of the AA course (N:13). All students were CS majors and were mostly juniors. On average, students attained $68 \%$ on the four multiple choice questions in the final exam. For the two design questions, students' average scores were $62 \%$ and $38 \%$. The second design question (with average score $38 \%$ ) involves writing a series of map and reduce tasks; and while many students provided a partially correct answer, only a few figured it out completely. These results indicate that the students were able to comprehend the concepts taught as part of the module and were able to answer both multiple choice and analytical questions successfully to a certain extent. After the rigorous lab session and many other individual troubleshooting sessions supported by the instructor and TAs, all students were able to perform Task 1 of the project, Task 2 was successfully completed by $85 \%$ of the students, and Task 3 was completed successfully by only $30 \%$ of the students. Overall, the student assessment results justify the module and show its effectiveness in achieving the proposed learning outcomes. Student assessment results of the multiple choice and analytical questions evidently supports students' in-depth understanding of the covered concepts (AA-LO1). The results of the first two 
project tasks show student competency in developing simple MapReduce applications (AA-LO2). The final task in the project exposed students to the cloud-enabled Spark environment and allowed them to understand runtime tradeoffs (AA-LO3); however, only a few students were able to complete this task.

\section{3 "Database Management" (DB) Module}

Currently, numerous application scenarios require processing very large datasets in a highly scalable and distributed fashion. Various types of big data systems have been designed to address this challenge and many of them have recognized the strengths of SQL as a query language. The proposed module for the introductory Database Management course is designed to expose students to the various types of big data systems and to integrate the study of SQL within these systems. This is a required course for both CS and IT majors at our institution and is typically attended by sophomore and junior students. The learning outcomes are

1. Recognize the key properties, strengths, and limitations of important big database management system (BDBMS) such as MapReduce, No-SQL, and New-SQL. (DB-LO1)

2. Develop hands-on experience in using SQL within Spark framework to load and query big datasets. (DB-LO2)

3.3.1 Lesson Plan. The first class (lecture) discusses limitations of relational database systems, and key properties, strengths, and limitations of various big data management systems such as MapReduce, No-SQL, and New-SQL. The second class starts with an introduction to the Spark distributed data processing framework, with Spark-SQL being a module within it for handling structed data processing. The lecture then introduces the students to the concept of DataFrames, emphasizing it can be created from a wide array of sources. The instructor then provides students with a tutorial and source code that instructs students the process of utilizing Jupyter notebook for developing and executing Spark-SQL applications. The tutorial provides instruction on creating DataFrames from JSON and CSV files, on manipulating them, and on running SQL queries programmatically within them.

3.3.2 Assessment Instruments A hands-on project and exam questions are utilized as assessment instruments. The exam contains four multiple choice questions that assess students' understanding of No-SQL and New-SQL systems and a detailed analytical question that assesses their comprehension of SparkSQL programming. The hands-on project is a progression of the tutorial and involves developing a Spark application that loads historical Facebook stock prices [17] and uses Spark SQL to query the data. The dataset is downloaded as a CSV file, where each record contains the stock values of a single date and includes the following attributes: date, open price, high price, low price, close price, volume, and adjClose (close price adjusted for dividends and splits). Students are asked to develop a Spark-SQL application that loads the file, creates a DataFrame based on the content, registers the DataFrame as a SQL temporary view, and then includes queries that show the answers to the following questions
1. Show all records that have gained value during daily transaction in the year 2018 (close $>=$ open).

2. Which day did Facebook stock gain maximum value?

3. Show the first 10 highest stock values.

4. Show the average sale volume for last 5 years.

5. Add two more questions and write queries to answer them. Students were taught to infer schema based on JSON and CSV input during the tutorials and the project requires them to perform similar inference while querying the Facebook data set. However, the project also contains a challenging part where the students are required to load and query a text file where schema should be enforced programmatically. Students are not explicitly taught the schema enforcement but are provided with some resources that they might find useful.

3.3.3 Assessment Results Overall, students were able to complete the project (infer schema automatically) while $63 \%$ of them attained A grade, $25 \%$ of them attained B grade and $12 \%$ attained $\mathrm{C}$ grade. The challenging part (define schema programmatically), however was successfully completed by only $12 \%$ of the students. Overall the students' quiz performance validates their thorough understanding of the key properties of the various BDBMS systems (DB-LO1) and their project grades reflect their competency in developing a Spark-SQL application in analyzing and querying big datasets (DB-LO2).

\subsection{Student Interest and Learning Experience}

A three-question student survey was designed to asses students' perceptions of their learning experiences and their level of confidence and interests and is administered after each intervention. The students provide their opinions about the three statements using a Likert scale of five values such as Strongly Agree, Agree, Neutral, Disagree and Strongly Disagree. The questions are as follows, where $\mathrm{X}$ refers to the module specific topics in each survey

- $\quad$ Q1 - I found the topics X interesting.

- $\quad$ Q2 - If a friend asks me what X are, I will be able to explain for 2-3 minutes.

- $\quad$ Q3 - I would like to learn more about X and would like to explore more in my future courses.

Fig. 2 shows the student survey results for the Computer Architecture course. It is evident from the results that the students positively attested the enhancements due to the module. More specifically, $79 \%$ of the students found (strongly agreed or agreed) the conveyed topics interesting, $72 \%$ of them strongly agreed or agreed that they are well-versed on the topics, and $86 \%$ reported their desire to learn the topics more after the intervention. Fig. 3 shows the student survey results for the Algorithm course and all (100\%) students found (strongly agreed or agreed) the conveyed topics interesting after the intervention. Similarly, $61 \%$ of the students strongly agreed or agreed that they are well-versed on the topics and $77 \%$ of the students expressed their eagerness to learn more. The survey results for the Database Management course is shown in Fig. 4 where $73 \%$ of the students attested (strongly agreed or agreed) the conveyed 
topics as interesting, $60 \%$ of the students strongly agreed or agreed that they are well-versed on the topics, and $87 \%$ of the students revealed their desire to learn the topics more. The relatively higher number of agreements with the self-reflection survey questions demonstrate that the students valued the experience, felt comfortable with the module content and grew further interest even though the duration of the intervention was very short.

\section{Conclusions}

The goal of this study is to explore the integration of big data and cloud computing modules into core undergraduate CS/IT courses and to evaluate its effectiveness. A substantial advantage of the modular approach is that a large number of CS/IT majors can be exposed to these contemporary topics and technologies via systematic and increasing integration throughout the computing curricula, and without the need of developing an additional core or elective course. This paper presents three such modules and our classroom experiences while deploying them. The student-generated evidence based on student performance and survey data supports our pedagogy, inspires us to continuously assess and update our interventions, and allows us to extend our interventions across multiple courses and semesters. The assessment results clearly show that the students were able to relate to the topics very well, found them to be interesting enough to explore and to retain, and developed significant interest and confidence after the interventions.

Development of this module content, lab and project-related artifacts such as tutorial, compiled programs, cloud instructions, input datasets, and assessment instruments required a large time investment. Instructors planning to reuse these modules must be well-prepared and organized in the classrooms to utilize their time in the best possible way. Our class size was rather small, and the instructors and their research assistants were able to support each student's needs in a timely manner. However, instructors who are planning to offer a similar module to a larger class must acquire enough TA resources for the duration of the module.

One limitation of the study is the lower number of students impacted by the modules and therefore, further repetitions of the interventions are paramount to make stronger conclusions about their effectiveness.

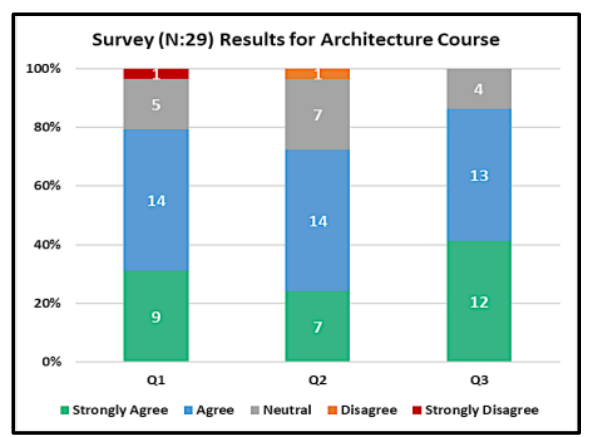

Figure 2: Students' Survey Results for CA Course.

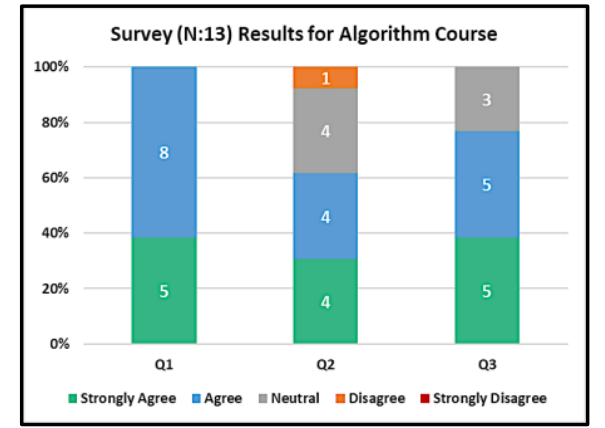

Figure 3: Students' Survey Results for AA Course.

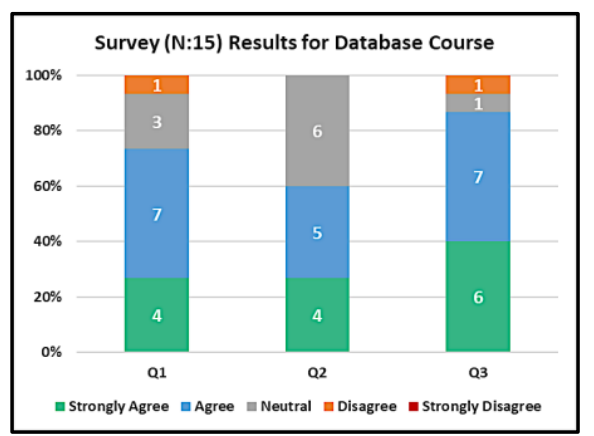

Figure 4: Students' Survey Results for DM Course.

In the future, we would like to perform research on more gradual and systematic integration of the developed modules across the curriculum, and research on assessing their collective effectiveness, rather than measuring the efficacy of a single module.

\section{ACKNOWLEDGMENTS}

This research was supported by National Science Foundation Award \# 1600864.

\section{REFERENCES}

[1] Joint Task Force on Computing Curricula, Association for Computing Machinery (ACM) and IEEE Computer Society. 2013. Computer Science Curricula, 2013.

[2] Criteria for accrediting computing programs, 2017-2018 ccreditation cycle http://www.abet.org/wp-content/uploads/2016/12/C001-17-18-CAC-Criteria10-29-16-1.pdf

[3] http://ibigcloud.altl.org/resources.html, 2018

[4] B. Ramamurthy, "A Practical and Sustainable Model for Learning and Teaching Data Science", Proceedings of the 47th ACM Technical Symposium on Computing Science Education (SIGCSE), March, 2016.

[5] S. J. Matthews, "Using Phoenix++ MapReduce to introduce undergraduate students to parallel computing", Journal of Computing Sciences in Colleges, vol. 32 Issue 6, June 2017.

[6] J. Eckroth, "Teaching Future Big Data Analysts: Curriculum and Experience Report", IEEE International Parallel and Distributed Processing Symposium Workshops (IPDPSW), May, 2017.

[7] A. S. Rabkin, C. Reiss, R. Katz, and D. Patterson. "Experiences teaching mapreduce in the cloud", In Proceedings of the 43rd ACM Technical Symposium on Computer Science Education (SIGCSE), 2012.

[8] M. S. Rehman, J. Boles, M. Hammoud, M. F. Sakr, "A Cloud Computing Course: From Systems to Services, Proceedings of the 46th ACM Technical Symposium on Computer Science Education (SIGCSE), 2015.

[9] CS5412: Cloud Computing, http://www.cs.cornell.edu/courses/cs5412/2018sp/ 
[10] CS309: Cloud Computing http://cs309a.stanford.edu/

[11] Center for Parallel and Distributed Computing Curriculum Development and Education resources (CDER), https:/grid.cs.gsu.edu/ tcpp/curriculum/

[12] System Stability Tester, http://systester.sourceforge.net/

[13] Phoronix Test Suite, https://www.phoronix-test-suite.com/

[14] Cloudera, Cloudera QuickStart VM

https://www.cloudera.com/downloads/quickstart_vms/5-12.html
[15] Chameleon cloud, https://www.chameleoncloud.org

[16] Wikipedia Page Views http://dumps.wikimedia.org/other/pagecounts-raw/

[17] Historial Facebook Stock Prices, https://finance.yahoo.com/quote/FB/history/ 